\title{
Infecção urinária em pacientes utilizando cateter vesical de demora internados em uma Unidade de Terapia Intensiva
}

\author{
Urinary infection in patients using delayed vesical catheter interned in an Intensive Care Unit \\ Infección urinaria en pacientes que utilizan catéter vesical de permanencia internos en \\ una Unidad de Cuidados Intensivos
}

Roberta Braga de Almeida1 ${ }^{1}$, Vera Lúcia Brahm dos Santos ${ }^{1}$, Maria Luiza Specht ${ }^{1}$, Lívia Souza da Rosa $^{2}$, Isadora Nunes Fernandes ${ }^{2}$, Pedro Borges de Souza ${ }^{2}$, Fernanda Pedrotti Moreira ${ }^{2 *}$.

\section{RESUMO}

Objetivo: Avaliar a prevalência de infecção do trato urinário (ITU) no setor de Unidade de Terapia Intensiva (UTI) de um hospital no sul do Brasil e sua associação com variáveis sociodemográficas e de saúde, incluindo a utilização de cateter vesical. Métodos: Estudo retrospectivo, transversal, incluindo pacientes adultos internados na UTI do Hospital Escola da Universidade Federal de Pelotas, no ano de 2019. A coleta de dados ocorreu através da revisão dos prontuários médicos do Serviço de Arquivo Médico e Estatístico. Resultados: Foram identificados 140 pacientes internados na UTI do hospital, apresentando $62,9 \%$ de prevalência ITU. Indivíduos que desenvolveram ITU permaneceram com cateter vesical por um tempo superior (média de 18,35 dias) do que aqueles que não desenvolveram $(\mathrm{p}<0,001)$. A Klebsiela pneumoniae foi observada em $46,6 \%$ dos exames, seguido pela Acinetobacter baumanii $(28,4 \%)$ e Escherichia Coli $(18,2 \%)$. Os principais antibióticos utilizados foram meropenem, piperacilina + tazobactan e polimixina B (33,0\%). Conclusão: Estes achados permitem contribuir na formulação de novas estratégias de intervenção, focando na atualização das práticas assistenciais e na educação continuada dos profissionais de saúde, reduzindo a prevalência desta infecção.

Palavras-chave: Infecção hospitalar, Unidades de terapia intensiva, Infecções urinárias, Cateter de demora.

\section{ABSTRACT}

Objective: To assess the prevalence of urinary tract infection (UTI) in the Intensive Care Unit (ICU) sector of a hospital in southern Brazil and its association with sociodemographic and health variables, including the use of a bladder catheter. Methods: Retrospective, cross-sectional study, including adult patients admitted to the ICU of the Teaching Hospital of the Federal University of Pelotas, in 2019. Data collection occurred through the review of the medical records of the Medical and Statistical Archive Service. Results: 140 patients admitted to the hospital's ICU were identified, with a $62.9 \%$ prevalence of UTI. Individuals who developed UTI remained with a bladder catheter for a longer time (mean of 18.35 days) than those who did not develop it $(\mathrm{p}<0.001)$. Klebsiela pneumoniae was observed in $46.6 \%$ of the exams, followed by Acinetobacter baumanii (28.4\%) and Escherichia Coli (18.2\%). The main antibiotics used were meropenem, piperacillin + tazobactan and polymyxin B (33.0\%). Conclusion: These findings make it possible to contribute to the formulation of new intervention strategies, focusing on updating care practices and continuing education for health professionals, reducing the prevalence of this infection.

Keywords: Cross infection, Intensive care units, Urinary tract infections, Catheters indwelling.

\section{RESUMEN}

Objetivo: Evaluar la prevalencia de infección del tracto urinario (ITU) en el sector Unidad de Cuidados Intensivos (UCI) de un hospital del sur de Brasil y su asociación con variables sociodemográficas y de salud, incluyendo el uso de sonda vesical. Métodos: Estudio retrospectivo, transversal, que incluyó a pacientes adultos ingresados en la UCI del $\mathrm{Hospital}$ Docente de la Universidad Federal de Pelotas, en el año 2019. La recolección de datos se realizó a través de la revisión de las historias clínicas del Servicio de Archivo Médico y Estadístico. Resultado: Se identificaron 140 pacientes ingresados en la UCl del hospital, con una prevalencia de ITU del 62,9\%. Los individuos que desarrollaron ITU permanecieron con un catéter vesical durante más tiempo (media de 18,35 días) que los que no la desarrollaron ( $p$ $<0,001)$. Se observó Klebsiela pneumoniae en el $46,6 \%$ de los exámenes, seguida de Acinetobacter baumanii $(28,4 \%)$ y Escherichia Coli $(18,2 \%)$. Los principales antibióticos utilizados fueron meropenem, piperacilina + tazobactan y polimixina B (33,0\%). Conclusión: Estos hallazgos permiten contribuir a la formulación de nuevas estrategias de intervención, enfocadas en la actualización de las prácticas de atención y educación continua de los profesionales de la salud, reduciendo la prevalencia de esta infección.

Palabras clave: Infección hospitalaria, Unidades de cuidados intensivos, Infecciones urinarias, Catéter de permanencia.

\footnotetext{
${ }^{1}$ Universidade Federal de Pelotas (UFPel), Pelotas - RS. *E-mail: fernanda.moreira@ucpel.edu.br
}

2 Universidade Católica de Pelotas (UCPel), Pelotas - RS. 


\section{INTRODUÇÃO}

As Infecções Relacionadas à Assistência à Saúde (IRAS) são reconhecidas como o evento adverso mais frequentemente relacionado aos cuidados em saúde (DUBBS SB e SOMMERKAMP SK, 2019). Geram consequências significativas para o sistema de saúde, são causa de taxas elevadas de mortalidade e morbidade, além de um grande ônus orçamentário às instituições, resultando em um maior tempo de internação e aumentos nos custos assistenciais, que pode ultrapassar em até três vezes em relação ao valor gasto com um paciente sem infecção (BRAGA IA, et al., 2018; SERRA-BURRIEL M, et al., 2020).

Entre as principais síndromes infecciosas estão as infecções primárias de corrente sanguínea, geralmente associadas a uso de cateter venoso central, pneumonia associada a ventilação mecânica e infecção do trato urinário (ITU), principalmente associada ao uso de cateter vesical de demora (CVD) (DUBBS SB e SOMMERKAMP SK, 2019).

No cenário mundial, as taxas de ocorrência de IRAS em países de baixa e média renda é de 15,5\%, enquanto, em países da Europa e Estados Unidos, estas ficam entre 4,5 e 7,1 (CASSINI A, et al., 2016). No Brasil, esta prevalência é de $10,8 \%$, porém a avaliação da magnitude das IRAS é considerada incipiente e localizada nas grandes capitais (FORTALEZA C, et al., 2017). Apesar das lacunas existentes, sabe-se que as IRAS estão entre as seis principais causas de óbito no Brasil, estando ao lado das doenças cardiovasculares, neoplasias, doenças respiratórias e infecciosas (MICHELIN AF e FONSECA MRCC, 2018).

Mundialmente, estima-se que quase meio milhão de casos de IRAS ocorre a cada ano nas Unidades de Terapia Intensiva (UTI). As especificidades desse setor o tornam um ambiente propício a infecções, incluindo a gravidade clínica dos pacientes, necessidade de internação prolongada, terapia com imunossupressores, uso rotineiro de antimicrobianos e consequente resistência à microrganismos, além do uso de procedimentos invasivos (MEDDINGS J, et al., 2020).

Dentre as IRAS destaca-se a ITU, representando aproximadamente 20 a $50 \%$ das notificações em UTI, e esta apresenta grande relação com a cateterização vesical pois, em aproximadamente $80 \%$ dos casos, essas infecções nosocomiais do trato urinário em UTI estão associadas ao uso de CVD (BARBOSA LR, et al., 2019; MOTA ÉC e OLIVEIRA AC, 2019). Esta infecção afeta qualquer parte do sistema urinário, incluindo uretra, bexiga, ureteres e rins e pode causar sequelas graves, incluindo recorrências frequentes, pielonefrite com sepse, dano renal e complicações causadas pelo uso constante ou repetitivo de antimicrobianos (DUBBS SB e SOMMERKAMP SK, 2019; FLORES-MIRELES AL, et al., 2015).

A infecção do trato urinário relacionada à cateter vesical de demora (ITU-RC) é considerada o tipo de IRAS mais comum. Estima-se que aproximadamente US $\$ 1.800,00$ são acrescidos nos custos de internação hospitalar devido as complicações e tempo de internação prolongado de pacientes que desenvolvem ITU através da inserção do CVD. Os riscos para ITU após a realização da inserção do CVD aumentam significativamente ao decorrer de 72 horas de permanência, o que ainda pode ser agravado caso ocorra trauma do tecido uretral durante a inserção (BARBOSA LR, et al., 2019).

As chances de o paciente desenvolver uma ITU-RC aumentam a medida que o cateterismo é prolongado, podendo aumentar $5 \%$ as chances a cada dia de uso do CVD, culminando em um risco de 100\% após 28 dias de cateterização (MOTA ÉC e OLIVEIRA AC, 2019).

Devido aos grandes riscos relacionados a cateterização, há a necessidade da adoção de estratégias para prevenir a ocorrência de ITU-RC (MOTA ÉC e OLIVEIRA AC, 2019). Estudos vêm mostrando que boa parte das ITU-RC poderiam ser evitadas ao adotar protocolos com enfoque no controle e prevenção destas infecções (ADVANI SD e FAKIH MG, 2019; ATKINS L, et al., 2020; MAJEED A, et al., 2019; MENEGUETI MG, et al., 2019; TYSON AF, et al., 2020). Evitando desta forma a subjetividade na indicação do uso de CVD e sua utilização por mais tempo que o necessário, reduzindo assim, a morbimortalidade destes pacientes, evitando-se até $69 \%$ dos agravos causados pela cateterização, porcentagem esta que corrobora em cerca de 380 mil infecções ITU-CV e 9 mil óbitos por ano (BARBOSA LR, et al., 2019; MOTA ÉC e OLIVEIRA AC, 2019). 
Desta forma, dada a complexidade da ocorrência de ITU, principalmente em ambiente de UTI, no intuito de desenvolver medidas de prevenção e controle, justifica-se a realização deste estudo, que, teve por objetivo avaliar a prevalência de ITU no setor de UTI de um hospital localizado no sul do Brasil, além da sua associação com variáveis sociodemográficas e de saúde, incluindo aquelas relacionadas com a utilização de CVD.

\section{MÉTODOS}

Trata-se de um estudo analítico, retrospectivo, do tipo transversal, incluindo pacientes adultos internados em uma UTI de um Hospital Escola localizado na cidade de Pelotas, Rio Grande do Sul durante o ano de 2019. A referida instituição é considerada centro de referência do Sistema Único de Saúde e atende pacientes de média complexidade, para a propedêutica, tratamento clínico e cirúrgico. O hospital tem capacidade total instalada de 164 leitos, dos quais seis são de UTI.

Foram incluídos neste estudo, pacientes com idade igual ou superior a 18 anos, internados na UTI do respectivo hospital. Os critérios de exclusão abrangeram pacientes com contagem de colônias em amostras de urina inferior a $105 \mathrm{UFC} / \mathrm{mL}$ e pacientes com resultado de culturas de urina não acompanhadas de antibiograma.

A coleta de dados ocorreu em novembro de 2020 por meio da identificação de todos os pacientes internados na UTI no ano de 2019. Os dados foram coletados por uma profissional de enfermagem, responsável pelo estudo, por meio da revisão dos prontuários médicos disponíveis no Serviço de Arquivo Médico e Estatístico (SAME) do hospital. Foram coletados, com um instrumento estruturado e elaborado para este estudo, variáveis demográficas e clínicas, além do resultado da urocultura e antibiograma, incluindo tipo de bactéria e antibiótico de escolha.

O desfecho do estudo foi a presença de ITU hospitalar. Será considerado ITU quando amostras de resultados alterados (ou seja, com quantidade elevada de leucócitos, bacteriúria moderada à intensa ou ainda para as amostras que apresentaram nitrito positivo) encaminhadas para urocultura apresentarem crescimento microbiano igual ou superior a $105 \mathrm{UFC} / \mathrm{mL}$.

Como variáveis de exposição, foram utilizadas: sexo (feminino; masculino), idade (coletada como variável numérica discreta), estado civil (solteiro(a); casado(a) ou vive com companheiro(a); separado(a); viúvo(a)), escolaridade (nunca estudou; até o primeiro grau; até o segundo grau; ensino superior), consumo atual de álcool e tabaco (não; sim), presença de comorbidades, sendo elas diabetes mellitus (não; sim), hipertensão arterial sistêmica (não; sim), neoplasia (não; sim) e vírus da imunodeficiência humana (HIV) (não; sim).

Também avaliou-se o uso de CVD (não; sim), indicação de uso de CVD (controle de diurese, paciente grave/instável, pós-operatório), tempo de permanência com CVD (coletada como variável numérica contínua), uso de antibióticos associados (não; sim), tipo de microrganismo encontrada no exame de urina (Fungos; Pseudomonas aeruginosa; Staphyloccocus ssp.; Proteus miriabilis; Escherichia coli; Acinetobacter baumanii e Klebsiella pneumoniae) e o antibiótico utilizado no tratamento de ITU (Meropenem; Piperacilina + tazobactan; Polimixina B; Vancomicina; Imipenem; Gentamicina; outros).

Todas os registros coletados foram transferidos para um banco de dados construído no EpiData® e, posteriormente, para o pacote estatístico SPSS $22.0 \AA$, onde foram realizadas as análises estatísticas. Após a análise das inconsistências, foi realizada análise descritiva da amostra estudada por meio de frequências absolutas e relativas e média e desvio padrão segundo variáveis demográficas, socioeconômicas e de saúde.

Teste Qui-quadrado e Teste T foram realizados para avaliar a relação entre ocorrência de ITU e variáveis demográficas, socioeconômicas de saúde e clínicas. Também se calculou a prevalência das principais bactérias encontradas nas uroculturas e os principais antibióticos utilizados no tratamento. $O$ nível de significância considerado para associações estatísticas foi de $p<0,05$.

A pesquisa foi aprovada pelo Comitê de Ética em Pesquisa da Universidade Católica de Pelotas (CAAE no 29719720.9.0000.5339), tendo sido garantido o compromisso de confidencialidade e sigilo para a coleta de dados. 


\section{RESULTADOS}

Durante os meses de janeiro a dezembro de 2019, foram identificados 140 prontuários de pacientes internados na UTI do hospital. De acordo com os resultados, que descrevem a amostra deste estudo, a maioria era do sexo feminino $(52,2 \%)$ e a média de idade dos pacientes foi de $58,4( \pm 16,5)$ anos. Um total de $49,1 \%$ era casado(a) ou vivia com companheiro(a) e $83,6 \%$ tinha o primeiro grau de escolaridade. O consumo de álcool foi verificado em $21,6 \%$ da amostra e o de tabaco em $38,6 \%$. Em relação às comorbidades, $25,9 \%$ tinha diabetes, 64,3\% tinha hipertensão, 46,0\% neoplasias e 14,3\% HIV. Em relação ao desfecho, a ocorrência de ITU foi observada em $62,9 \%$ dos pacientes internados (Tabela 1).

Tabela 1 - Descrição da amostra segundo variáveis sociodemográficas e de saúde de pacientes internados na UTI durante os meses de janeiro a dezembro de 2019.

\begin{tabular}{|c|c|c|}
\hline \multirow{2}{*}{$\begin{array}{ll} & \text { Variável } \\
\text { Sexo } & \end{array}$} & \multicolumn{2}{|c|}{ Dados descritivos } \\
\hline & & \\
\hline Feminino & 71 & 52,2 \\
\hline Masculino & 65 & 47,8 \\
\hline Idade $^{\mathrm{b}}$ & 58,4 & $\pm 16,5$ \\
\hline \multicolumn{3}{|l|}{ Estado civila } \\
\hline Solteiro(a) & 36 & 32,7 \\
\hline Casado(a)/vive com companheiro(a) & 54 & 49,1 \\
\hline Separado(a) & 8 & 7,3 \\
\hline Viúvo(a) & 12 & 10,9 \\
\hline \multicolumn{3}{|l|}{ Escolaridade $^{a}$} \\
\hline Nunca estudou & 10 & 7,8 \\
\hline Primeiro grau & 107 & 83,6 \\
\hline Segundo grau & 7 & 5,5 \\
\hline Nível superior & 4 & 3,1 \\
\hline \multicolumn{3}{|l|}{ Consumo de álcoola } \\
\hline Sim & 27 & 21,6 \\
\hline Não & 98 & 78,4 \\
\hline \multicolumn{3}{|l|}{ Consumo de tabaco $^{a}$} \\
\hline Sim & 54 & 38,6 \\
\hline Não & 86 & 61,4 \\
\hline \multicolumn{3}{|l|}{ Diabetes Melittus ${ }^{\mathrm{a}}$} \\
\hline Sim & 36 & 25,9 \\
\hline Não & 103 & 74,1 \\
\hline \multicolumn{3}{|l|}{ Hipertensão Arterial Sistêmica (HAS) ${ }^{a}$} \\
\hline Sim & 90 & 64,3 \\
\hline Não & 50 & 35,7 \\
\hline \multicolumn{3}{|l|}{ Neoplasias $^{a}$} \\
\hline Sim & 64 & 46,0 \\
\hline Não & 75 & 54,0 \\
\hline \multicolumn{3}{|l|}{ Vírus da imunodeficiência humana (HIV) ${ }^{a}$} \\
\hline $\operatorname{Sim}$ & 20 & 14,3 \\
\hline Não & 120 & 85,7 \\
\hline \multicolumn{3}{|l|}{ Infecção do Trato Urinário (ITU)a } \\
\hline Sim & 88 & 62,9 \\
\hline Não & 52 & 37,1 \\
\hline TOTAL & 140 & 100,0 \\
\hline
\end{tabular}

Legenda: a: dados representados por frequência absoluta e frequência relativa; ${ }^{b}$ : dados representados por média e desvio padrão. Fonte: Almeida RB, et al., 2021.

Os resultados apresentados a seguir descrevem a associação entre variáveis sociodemográficas e de saúde e ocorrência de ITU nos pacientes internados. Não foram observadas associações estatisticamente significativas, mas observa-se uma tendência de maior ocorrência de ITU em pacientes com consumo de álcool e tabaco e aqueles com diabetes mellitus e hipertensão arterial (Tabela 2). 
Tabela 2 - Associação entre variáveis sociodemográficas e de saúde e ocorrência de ITU em pacientes internados na UTI.

\begin{tabular}{|c|c|c|c|}
\hline \multirow{2}{*}{ Variável } & \multicolumn{2}{|c|}{ ITU } & \multirow{2}{*}{ Valor-p } \\
\hline & Sim & Não & \\
\hline Sexo $^{a}$ & & & 0,690 \\
\hline Feminino & $46(54,1)$ & $25(49,0)$ & \\
\hline Masculino & $39(45,9)$ & $26(51,0)$ & \\
\hline Idade $^{b}$ & $59,76 \pm 16,28$ & $55,98 \pm 16,73$ & 0,194 \\
\hline Escolaridade $^{a}$ & & & 0,179 \\
\hline Nunca estudou & $8(10,0)$ & $2(4,2)$ & \\
\hline Primeiro grau & $68(85,0)$ & $39(81,2)$ & \\
\hline Segundo grau & $3(3,7)$ & $4(8,3)$ & \\
\hline Nível superior & $1(1,3)$ & $3(6,3)$ & \\
\hline Estado civila & & & 0,872 \\
\hline Solteiro(a) & $20(31,2)$ & $16(34,8)$ & \\
\hline Casado(a)/vive com companheiro(a) & $32(50,0)$ & $22(47,8)$ & \\
\hline Separado(a) & $4(6,3)$ & $4(8,7)$ & \\
\hline Viúvo(a) & $8(12,5)$ & $4(8,7)$ & \\
\hline Consumo de álcool ${ }^{\mathrm{a}}$ & $21(26,3)$ & $6(13,3)$ & 0,145 \\
\hline Consumo de tabaco $^{a}$ & $38(43,2)$ & $16(30,8)$ & 0,210 \\
\hline Diabetes Melittus ${ }^{\mathrm{a}}$ & $26(29,9)$ & $10(19,2)$ & 0,235 \\
\hline Hipertensão Arterial Sistêmica (HAS) ${ }^{a}$ & $58(65,9)$ & $32(35,6)$ & 0,735 \\
\hline Neoplasias $^{\mathrm{a}}$ & $40(45,5)$ & $24(47,1)$ & 0,855 \\
\hline Vírus da imunodeficiência humana (HIV) ${ }^{a}$ & $14(15,9)$ & $6(11,5)$ & 0,643 \\
\hline
\end{tabular}

Legenda: ${ }^{a}$ : dados representados por frequência absoluta e frequência relativa; ${ }^{\text {b: }}$ dados representados por média e desvio padrão. Fonte: Almeida RB, et al., 2021.

Em relação aos resultados que apresentam a associação entre variáveis clínicas e ocorrência de ITU, observa-se que indivíduos que desenvolveram ITU permaneceram com CV por um tempo expressivamente superior (18,4 $\pm 15,8$ dias) do que aqueles que não desenvolveram (7,0 $\pm 9,7$ dias) $(p<0,001)$. Apesar de não ter observado associação, a ocorrência de ITU mostra-se superior entre aqueles indivíduos com indicação de uso de CV devido a ser um paciente grave/instável (70,4\% vs. 29,6\%) e devido ao pós-operatório (60,0\% vs. 40,0\%) (Tabela 3).

Tabela 3 - Associação entre variáveis clínicas e ocorrência de ITU em pacientes internados na UTI.

\begin{tabular}{|c|c|c|c|}
\hline \multirow{2}{*}{ Variável } & \multicolumn{2}{|c|}{ ITU } & \multirow{2}{*}{ Valor-p } \\
\hline & Sim & Não & \\
\hline Uso de cateter vesical de demora ${ }^{a}$ & & & 0,690 \\
\hline Sim & $75(63,6)$ & $43(35,4)$ & \\
\hline Não & $13(59,1)$ & $9(40,9)$ & \\
\hline Indicação do uso de cateter ${ }^{a}$ & & & 0,055 \\
\hline Controle de diurese & $9(45,0)$ & $11(55,0)$ & \\
\hline Paciente grave/instável & $57(70,4)$ & $24(29,6)$ & \\
\hline Pós-operatório & $21(60,0)$ & $14(40,0)$ & \\
\hline Tempo de permanência com o cateter ${ }^{b}$ & $18,4 \pm 15,8$ & $7,0 \pm 9,7$ & $<0,001$ \\
\hline Uso de antibióticos associados ${ }^{a}$ & & & 0,676 \\
\hline Sim & $80(97,6)$ & $2(2,4)$ & \\
\hline Não & $7(100,0)$ & - & \\
\hline
\end{tabular}

Legenda: a: dados representados por frequência absoluta e frequência relativa; b: dados representados por média e desvio padrão. Fonte: Almeida RB, et al., 2021. 
Todos 88 pacientes com ITU realizaram urocultura. Em relação ao tipo e bactéria encontrada no exame, a Klebsiela pneumoniae foi observada em $46,6 \%$ dos exames, seguido pela Acinetobacter baumanii $(28,4 \%)$ e Escherichia coli (18,2\%) (Figura 1). Dez pacientes apresentaram mais de uma bactéria na urocultura, sendo que, em oito casos foi observado K. pneumoniae e $A$. baumanii $(9,1 \%)$ e em dois casos, $P$. miriabilis e $A$. baumanii (2,3\%) do total (dados não apresentados).

Figura 1 - Descrição das bactérias identificadas nos exames de urocultura de pacientes internados em UTI.

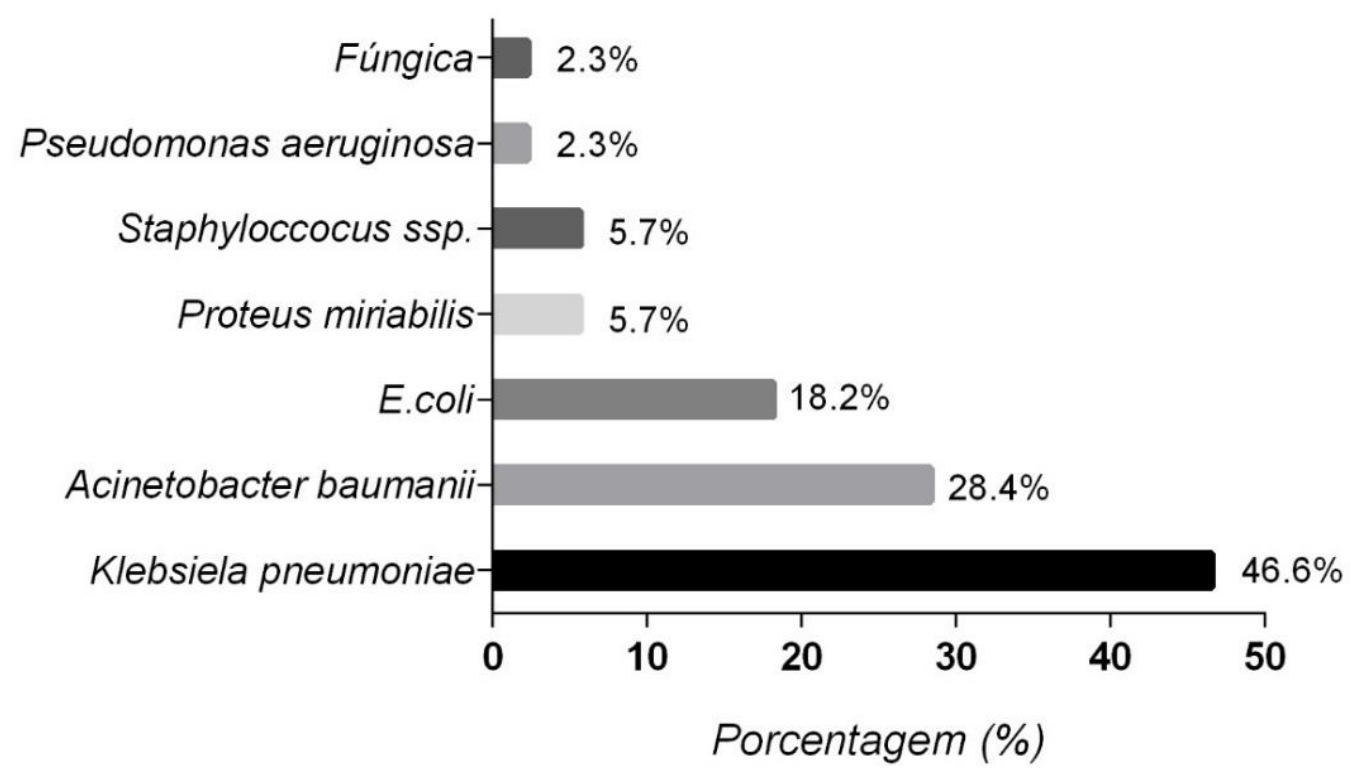

Fonte: Almeida RB, et al., 2021.

O principal antibiótico de escolha no tratamento de ITU nos pacientes internados em UTI foi o meropenem $(83,0 \%)$ seguido por piperacilina + tazobactan $(59,1 \%)$ e por polimixina $B(33,0 \%)$. A principal associação de antibióticos foi meropenem+pipetazo em $50 \%(n=44)$ dos pacientes com ITU (Figura 2).

Figura 2 - Descrição dos antibióticos utilizados para tratamento de ITU de pacientes internados em UTI.

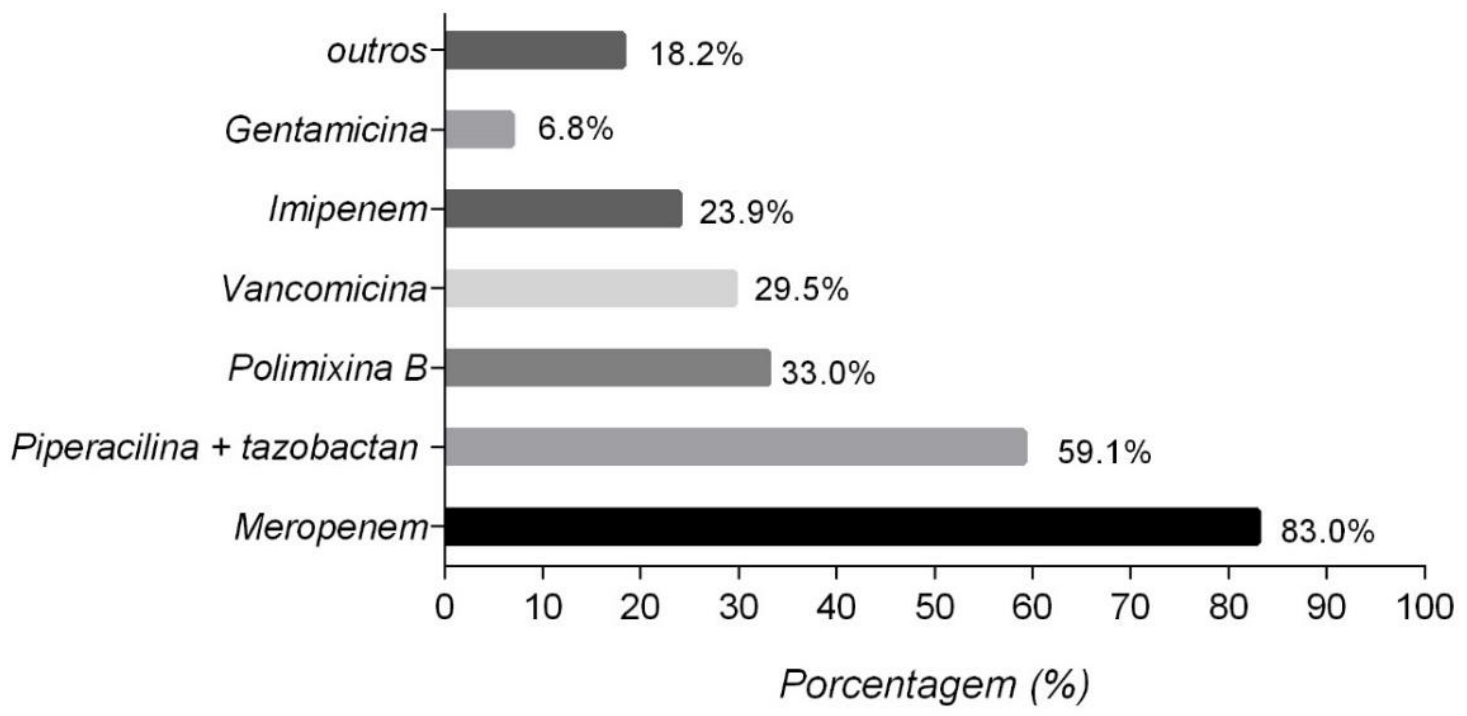

Fonte: Almeida RB, et al., 2021. 


\section{DISCUSSÃO}

O presente estudo identificou que a ocorrência de ITU foi observada em $62,9 \%$ dos pacientes internados e associou-se com tempo de permanência com CVD. As bactérias mais frequentemente encontradas nas uroculturas foram K. pneumoniae, A. baumanii e E. coli, enquanto os antibióticos mais utilizados no tratamento foram meropenem, seguido por piperacilina + tazobactan e polimixina $B$.

A prevalência de ITU identificada neste estudo encontra-se acima do que descreve a literatura. Em ambiente de UTI, a maioria dos estudos refere uma prevalência de cerca de 30\% (MOTA ÉC e OLIVEIRA AC, 2019). Outros estudos identificam valores próximos a 40\% (FLORES-MIRELES AL, et al., 2015), porém, ainda assim, inferior ao observado. Neste sentido, atualização das práticas assistenciais dos profissionais de saúde, com processos de educação continuada, elaboração de protocolos e padronização dos procedimentos em ambientes de atenção à saúde pode ser uma alternativa, com vistas na redução da ocorrência deste tipo de infecção (ADVANI SD e FAKIH MG, 2019; ATKINS L, et al., 2020; MAJEED A, et al., 2019; MENEGUETI MG, et al., 2019; TYSON AF, et al., 2020).

A maioria dos pacientes com ITU fizeram uso de CVD e, o tempo de permanência associou-se com a sua ocorrência, onde indivíduos com ITU permaneceram com cateter por tempo superior àqueles sem ITU. As ITU-RC foram responsáveis por $63,6 \%$ dos casos de ITU, o que vem de encontro com a literatura pois, aproximadamente $80 \%$ das ITU relacionadas à assistência à saúde estão associadas ao CVD. Neste sentido, o risco de desenvolver ITU-RC aumenta com a duração do cateterismo, podendo aumentar $5 \%$ a cada dia de uso. Assim, como já relatado em outros estudos, estima-se que, após 28 dias de cateterização, esse risco eleva-se para $100 \%$, culminando em aproximadamente $4 \%$ dos pacientes apresentando uma evolução para sepse secundária à ITU, com uma taxa de mortalidade estimada em até 30\% (MOTA ÉC e OLIVEIRA AC, 2019).

O cateterismo vesical é uma técnica invasiva onde é introduzida uma sonda através da uretra até a bexiga com a finalidade de drenar a urina, sendo indicado para paciente com grave lesão medular, politraumatizado, controle de volume em débito urinário, conforto para os pacientes terminais, pacientes com obstrução do trato urinário, pós-operatório de cirurgias urológicas, ortopédicas e ginecológicas (CARDOSO SAC e MAIA LFDS, 2014).

Várias estratégias para prevenir a ocorrência de ITU-RC têm sido preconizadas, e todas apontam um princípio fundamental: indicação restrita, de acordo com recomendações de diretrizes, inserção com técnica asséptica, cuidados na manutenção e rigor, principalmente, quanto ao tempo de permanência. Limitar o uso de cateteres urinários para pacientes com indicação clara e removê-los assim que não forem mais necessários são consideradas as medidas de prevenção mais eficazes e econômicas. Apesar dessas recomendações, o uso do CVD ainda é considerado variável, com pouco ou nenhum controle, repercutindo em altas taxas de ITU-RC (FLORES-MIRELES A, et al., 2019).

Estudos estimam que através da adoção de protocolos que tenham enfoque no controle e prevenção destas infecções, muitas das ITU-RC são evitáveis (ADVANI SD e FAKIH MG, 2019; ATKINS L, et al., 2020; MAJEED A, et al., 2019; MENEGUETI MG, et al., 2019; TYSON AF, et al., 2020), ressaltando a importância de uma equipe multidisciplinar consistente, capaz de realizar intervenções oportunas, além de suceder a uma avaliação do paciente, possibilitando intervenções de forma a evitar o uso errôneo do CVD (BARBOSA LR, et al., 2019).

Além dos cuidados com instalação e manipulação do CVD, sabe-se que existem fatores de risco associados ao paciente que podem potencializar sua ocorrência, incluindo idade avançada, sexo feminino, comorbidades como diabetes mellitus, condições clínicas gerais do paciente, insuficiência renal crônica, alterações no estado nutricional, imunocomprometimento e anomalias anatômicas no trato urinário (FLORESMIRELES AL, et al., 2015). É preconizado minimizar o uso e a duração do CVD sempre que possível em todos os pacientes, todavia deve-se haver uma maior preocupação com os pacientes que são considerados como grupo de maior predisposição a ITU-RC, levando em consideração os fatores clínicos e sociodemográficos dos mesmos (GOULD CV, et al., 2010; MOTA ÉC e OLIVEIRA AC, 2019). Apesar disso, o presente estudo não identificou fatores sociodemográficos e de saúde associados à ocorrência de ITU. 
Todavia, estudos mostraram relação entre as ITUs Relacionadas à Assistência em Saúde e fatores de risco, sendo o uso de antimicrobianos no último mês, ITU no ano anterior e diabetes, além de transplante renal e pré-existência de alteração urológica (BAENAS DF, et al., 2018). Em outros estudos, pode-se encontraram-se resultados somente associados ao quadro clínico, quando observada a utilização de CVD, constatou-se que a utilização de antimicrobianos no último mês e dias de internação foram maiores nos pacientes ITU-RC (BAENAS DF, et al., 2018; MOTA ÉC e OLIVEIRA AC, 2019).

Os três microrganismos mais observados nos exames de urocultura foram K. pneumoniae, A. baumanii e E. coli, respectivamente. A literatura destaca os bacilos gram-negativos como os agentes etiológicos mais comumente associados a ITU (BARBOSA LR, et al., 2019). Neste sentido, estudos nacionais e internacionais identificam a Escherichia Coli como o principal agente etiológico dessas infecções (BARBOSA LR, et al., 2019; DAVIS C, 2019). Outros microrganismos também estão fortemente associados, como E. coli, Enterococcus spp., K. pneumoniae, Pseudomonas aeruginosa, Staphylococcus spp. Ainda, o aparecimento de fungos, como a Candida sp também é frequentemente observado (SABIH A e LESLIE SW, 2021).

Os resultados apresentados pelas uroculturas do presente estudo, diferente com o relatado pela maior parte da literatura, apresentam a K. pneumoniae como o principal agente identificado nos pacientes com ITU, e não E. coli como relatado por outros estudos (BARBOSA LR, et al., 2019; DAVIS C, 2019; FLORESMIRELES A, et al., 2019). Todavia, o resultado obtido em nosso estudo vai ao encontro do resultado obtido por Tavares JMDM, et al. (2020), onde o mesmo encontrou a $K$. pneumoniae, como principal agente bacteriano $(21,9 \%)$ e a E. coli como o segundo, relacionado a ITU-RC nesses pacientes. A K. pneumoniae está constantemente associada a ITU, independente da presença de CVD pois, esta bactéria possui mecanismos especializados, voltados para a colonização e invasão das células presentes na bexiga (FLORES-MIRELES A, et al., 2019).

A maioria das infecções em pacientes com cateterização por curto período de tempo é causada por um único microrganismo. Já infecções que ocorrem em pacientes necessitando de uso prolongado de CVD, são frequentemente polimicrobianas (LEARMAN BS, et al., 2019). Infecções polimicrobianas foram observadas em dez pacientes, com o A. baumanii como um dos agentes em todos os casos.

Um resultado preocupante encontrado no estudo foi a alta prevalência de $A$. baumannii $(28,4 \%)$ nestes pacientes. Este patógeno nosocomial vem ganhando atenção da comunidade científica pois está associado a infecções hospitalares, sendo responsável por aproximadamente $9,4 \%$ de infecções em UTIs (VINCENT $\mathrm{JL}$, et al., 2020). Ademais, devido a seus fatores de virulência e resistência aos antimicrobianos, a Organização Mundial de Saúde, em 2017, o elencou como patógeno prioritário no desenvolvimento de novos antimicrobianos, o que ressalta ainda mais a importância do controle das infecções por este patógeno (WHO, 2017).

O principal antibiótico de escolha no tratamento de ITU nos pacientes internados em UTI foi o meropenem, seguido por piperacilina + tazobactan e por polimixina B. De acordo com alguns estudos, o uso de antibióticos é aproximadamente dez vezes maior em UTI do que em enfermarias dos hospitais (BRAGA IA, et al., 2018). $O$ uso indiscriminado de antimicrobianos é considerado um dos principais riscos para a seleção de bactérias multirresistentes (PEREIRA JQ, et al., 2018). A literatura descreve que os carbapênemicos, vancomicina e cefalosporinas de amplo espectro são prescritos com muita frequência entre os países da América Latina. No Brasil, a alta densidade de uso de antimicrobianos é ainda mais significativa, principalmente pelo uso excessivo de antibióticos das classes $\beta$-lactâmicos, incluindo os carbapênemicos e fluorquinolonas (DANTAS RCC, et al., 2017), conforme observado no presente estudo.

Esta exposição excessiva aos antimicrobianos, sendo estes administrados de forma empírica, permite o crescimento excessivo de patógenos com resistência intrínseca ou adquirida ao medicamento administrado (LEE YC, et al., 2016; TIMSIT JF, et al., 2019). A preocupação com as altas taxas de resistência e a administração empírica de medicamentos mostra-se evidente no estudo de Lee YC, et al. (2016) onde pacientes que apresentavam bactérias multidroga resistentes (MDR), receberam tratamentos ineficazes, quando comparados a pacientes que apresentavam bactérias não resistentes. Ademais, cabe ressaltar que, 
no mesmo estudo, observou-se altas taxas de resistência apresentaram associação com a necessidade da utilização de CVD, o que implica em possíveis tratamentos ineficazes para estes pacientes, provocando um amento na morbimortalidade dos mesmos (LEE YC, et al., 2016; TIMSIT JF, et al., 2019).

Esta constatação reflete a necessidade de um maior conhecimento epidemiológico local, a fim de evitar tratamentos errôneos, mesmo que empíricos. A necessidade da administração do antibiótico correto é de extrema importância para o paciente, todavia, alguns estudos observaram que a mortalidade hospitalar em pacientes com ITU causada por patógenos MDR foi mais elevada, ressaltando a importância de um tratamento eficaz. Isto torna-se ainda mais preocupante, uma vez que a permanência do paciente em UTI, além do uso de CVD, corrobora para o surgimento de ITU, podendo esta ser devido a um patógeno MDR, agravando o quadro clínico do paciente (LEE YC, et al., 2016; MOTA ÉC e OLIVEIRA AC, 2019; TIMSIT JF, et al., 2019).

O presente estudo apresenta algumas limitações. Uma delas refere-se à validade externa dos resultados, devido ao fato de que este estudo se limita a um único serviço de saúde, desta forma, a generalização dos achados para a população geral fica comprometida. Outra questão refere-se ao tamanho de amostra, que ficou abaixo do esperado e pode ter resultando em baixo poder estatístico das associações observadas. $O$ estudo também apresenta pontos positivos, pois permitiu conhecer a realidade do serviço de saúde em questão, e, a partir de então, será possível desenvolver estratégias para melhoria deste cenário.

\section{CONCLUSÃO}

O presente estudo identificou que três a cada cinco pacientes internados em UTI desenvolveram ITU e esta, associou-se com o tempo de permanência com CVD, ademais, Klebsiela pneumoniae, Acinetobacter baumanii e Escherichia coli foram os microrganismos mais frequentemente identificados nos exames de urocultura. O estudo também permitiu conhecer a ocorrência deste desfecho em um setor de grande importância de um hospital que é a UTI, de forma que seja possível contribuir na formulação de novas estratégias de intervenção, principalmente com foco na atualização das práticas assistenciais e na educação continuada dos profissionais de saúde objetivando a redução da prevalência desta infecção.

\section{AGRADECIMENTOS}

Ao Hospital Escola da Universidade Federal de Pelotas, pela oportunidade de realização desta pesquisa.

\section{REFERÊNCIAS}

1. ADVANI SD, FAKIH MG. The evolution of catheter-associated urinary tract infection (CAUTI): Is it time for more inclusive metrics? Infection Control \& Hospital Epidemiology, 2019; 40(6): 681-685.

2. ATKINS L, et al. Reducing catheter-associated urinary tract infections: a systematic review of barriers and facilitators and strategic behavioural analysis of interventions. Implementation Science, 2020; 15(1): 1-22.

3. BAENAS DF, et al. Nosocomial urinary tract infection: an analysis beyond urinary catheterization. Revista Chilena de Infectología, 2018; 35(3): 246-252.

4. BARBOSA LR, et al. Infecção do trato urinário associada ao cateter vesical em uma unidade de terapia intensiva. Revista de Epidemiologia e Controle de Infecção, 2019; 9(2): 103-108.

5. BRAGA IA, et al. Multi-hospital point prevalence study of healthcare-associated infections in 28 adult intensive care units in Brazil. Journal of Hospital Infection, 2018; 99(3): 318-324.

6. CARDOSO SAC, MAIA LFDS. Cateterismo vesical de demora na UTI adulto: o papel do enfermeiro na prevenção de infecção do trato urinário. Revista Recien, 2014; 4(12): 5-14.

7. CASSINI A, et al. Burden of Six Healthcare-Associated Infections on European Population Health: Estimating Incidence-Based Disability-Adjusted Life Years through a Population Prevalence-Based Modelling Study. PLoS Medicine, 2016; 13(10): e1002150.

8. DANTAS RCC, et al. Molecular epidemiological survey of bacteremia by multidrug resistant Pseudomonas aeruginosa: the relevance of intrinsic resistance mechanisms. PLoS One, 2017; 12(5): e0176774.

9. DAVIS C. Catheter-associated urinary tract infection: signs, diagnosis, prevention. British Journal of Nursing, 2019; 28(2): 96-100. 
10. DUBBS SB, SOMMERKAMP SK. Evaluation and Management of Urinary Tract Infection in the Emergency Department. Emergency Medicine Clinics of North America, 2019; 37(4): 707-723.

11. FLORES-MIRELES A, et al. Pathophysiology, Treatment, and Prevention of Catheter-Associated Urinary Tract Infection. Topics in Spinal Cord Injury Rehabilitation, 2019; 25(3): 228-240.

12. FLORES-MIRELES AL, et al. Urinary tract infections: epidemiology, mechanisms of infection and treatment options. Nature Reviews Microbiology, 2015; 13(5): 269-284.

13. FORTALEZA C, et al. Multi-state survey of healthcare-associated infections in acute care hospitals in Brazil. Journal of Hospital Infection, 2017; 96(2): 139-144.

14. GOULD CV, et al. Guideline for prevention of catheter-associated urinary tract infections 2009. Infection Control \& Hospital Epidemiology, 2010; 31(4): 319-326.

15. LEARMAN BS, et al. A Rare Opportunist, Morganella morganii, Decreases Severity of Polymicrobial CatheterAssociated Urinary Tract Infection. Infection and Immunity, 2019; 88(1): e00691-19.

16. LEE YC, et al. Bacteremic Urinary Tract Infection Caused by Multidrug-Resistant Enterobacteriaceae Are Associated With Severe Sepsis at Admission: Implication for Empirical Therapy. Medicine (Baltimore), 2016; 95(20): e3694.

17. MAJEED A, et al. Does antimicrobial coating and impregnation of urinary catheters prevent catheter-associated urinary tract infection? A review of clinical and preclinical studies. Expert Review of Medical Devices, 2019; 16(9): 809-820.

18. MEDDINGS J, et al. Multistate programme to reduce catheter-associated infections in intensive care units with elevated infection rates. BMJ Quality \& Safety, 2020; 29(5): 418-429.

19. MENEGUETI MG, et al. Long-term prevention of catheter-associated urinary tract infections among critically ill patients through the implementation of an educational program and a daily checklist for maintenance of indwelling urinary catheters: A quasi-experimental study. Medicine (Baltimore), 2019; 98(8): e14417.

20. MICHELIN AF, FONSECA MRCCd. Perfil epidemiológico das infecções hospitalares na unidade de terapia intensiva de um hospital terciário. Nursing (São Paulo), 2018; 2037-2041.

21. MOTA ÉC, OLIVEIRA AC. Catheter-associated urinary tract infection: why do not we control this adverse event? A Revista da Escola de Enfermagem da USP, 2019; 53: e03452.

22. PEREIRA JQ, et al. Use of antibiotics by adults: a population-based cross-sectional study. Sao Paulo Medical Journal, 2018; 136(5): 407-413.

23. SABIH A, LESLIE SW. Complicated Urinary Tract Infections. In: StatPearls [internet], 2021.

24. SERRA-BURRIEL M, et al. Impact of multi-drug resistant bacteria on economic and clinical outcomes of healthcareassociated infections in adults: Systematic review and meta-analysis. PLoS One, 2020; 15(1): e0227139.

25. TAVARES JMDM, et al. Incidência de infecção urinária em pacientes hospitalizados em uso de cateter vesical de demora. Revista Eletrônica Acervo Saúde, 2020; 12(8): e3497.

26. TIMSIT JF, et al. Rationalizing antimicrobial therapy in the ICU: a narrative review. Intensive Care Medicine, 2019; 45(2): 172-189.

27. TYSON AF, et al. Implementation of a Nurse-Driven Protocol for Catheter Removal to Decrease Catheter-Associated Urinary Tract Infection Rate in a Surgical Trauma ICU. Journal of Intensive Care Medicine, 2020; 35(8): 738-744.

28. VINCENT JL, et al. Prevalence and Outcomes of Infection Among Patients in Intensive Care Units in 2017. Journal of the American Medical Association, 2020; 323(15): 1478-1487.

29. WHO. WORLD HEALTH ORGANIZATION. WHO publishes list of bacteria for which new antibiotics are urgently needed. 2017. Disponível em: https://www.who.int/news/item/27-02-2017-who-publishes-list-of-bacteria-for-whichnew-antibiotics-are-urgently-needed. Acesso em: 1 mai 2021. 